\title{
Review of Development of the Smooth Particle Hydrodynamics (SPH) Method
}

\author{
Rade Vignjevic \\ Crashworthiness, Impact and Structural Mechanics (CISM) \\ Cranfield University, UK
}

\begin{abstract}
The paper gives an overview of developments of the SPH method. Especial attention is given to the main shortcomings of the original form of the method namely consistency, tensile instability and zero energy modes. An example of derivation of correction necessary to assure first order consistency is given. The origin of the tensile instability and few proposed solutions to this problem are described. Similar consideration is given with respect to the zero energy modes typical for the collocational SPH method.
\end{abstract}

\section{Introduction}

This paper discusses the development of the Smooth Particle Hydrodynamics (SPH) method in its original form based on updated Lagrangian formalism. SPH is a relatively new numerical technique for the approximate integration of partial differential equations. It is a meshless Lagrangian method that uses a pseudoparticle interpolation method to compute smooth field variables. Each pseudoparticle has a mass, Lagrangian position, Lagrangian velocity, and internal energy; other quantities are derived by interpolation or from constitutive relations.

The advantage of the meshless approach is its ability to solve problems that cannot be effectively solved using other numerical techniques. It does not suffer from the mesh distortion problems that limit Lagrangian approaches based on structured mesh when simulating large deformations. As it is a Lagrangian method it naturally tracks material history information, such as damage, without the diffusion that occurs in Eulerian approaches due to advection.

Gingold [14] and Lucy [28] initially developed SPH in 1977 for the simulation of astrophysics problems. Their breakthrough was a method for the calculation of derivatives that did not require a structured computational mesh. Review papers by Benz [8] and Monaghan [31] cover the early development of SPH. Libersky and Petchek [26] extended SPH to work with the full stress tensor in 2D. This addition allowed SPH to be used in problems where material strength is important. The development SPH with strength of materials continued with extension to 3D [27], and the linking of SPH with existing finite element codes [3, 19].

The introduction of material strength highlighted shortcomings in the basic method: accuracy, tensile instability, zero energy modes and artificial viscosity. These shortcomings were identified in a first comprehensive analysis of the SPH method 
by Swegle [45], and Wen [55]. The problems of consistency and accuracy of the SPH method, identified by Belytschko [5], were addressed by Randles and Libersky [41] and Vignjevic and Campbell [51]. This resulted in a normalised first order consistent version of the SPH method with improved accuracy. The attempts to ensure first order consistency in SPH led to the development of a number of variants of the SPH method, such as Element Free Galerkin Mehod (EFGM) [6, 23], Reproducing Kernel Particle Method (RKPM) [56, 57], Moving Least Square Particle Hydrodynamics (MLSPH) [12], Meshless Local Petrov Galerkin Method (MLPG) [1]. These methods allow the restoration of consistency of any order by means of a correction function. It has been shown by Atluri $[1,2]$ that the approximations based on corrected kernels are identical to moving least square approximations. The issue of stability was dealt with in the context of particle methods in general by Belytschko [7], and independently by Randles [42]. They reached the same conclusions as Swegle [45] in his initial study.

In spite of these improvements, the crucial issue of convergence in a rigorous mathematical sense and the links with conservation have not been well understood. Encouraging preliminary steps in this direction have already been put forward very recently by Ben Moussa [38], who proved convergence of their meshless scheme for non-linear scalar conservation laws; see also [39]. This theoretical result appears to be the first of its kind in the context of meshless methods. Furthermore, Ben Moussa proposed an interesting new way to stabilise normalised SPH and allow for treatment of boundary conditions by incorporating upwinding, an approach usually associated with finite volume shock-capturing schemes of the Godunov type, see $[47,48,49]$. The task of designing practical schemes along these lines is pending, and there is scope for cross-fertilisation between engineers and mathematicians and between SHP specialists and Godunov-type schemes specialists.

The improvements of the methods in accuracy and stability achieved by kernel renormalisation or correction, have not, however, come for free; now it is necessary to treat the essential boundary conditions in a rigorous way. The approximations in SPH do not have the property of strict interpolants so that in general they are not equal to the particle value of the dependent variable, i.e. $u^{h}\left(x_{j}\right)=\sum_{I} \phi_{I}\left(x_{j}\right) u_{I} \neq u_{J}$. Consequently it does not suffice to impose zero values for $u_{I}$ at the boundary positions to enforce homogeneous boundary conditions.

The treatment of boundary conditions and contact was neglected in the conventional SPH method. If the imposition of the free surface boundary condition (stress free condition) is simply ignored, then conventional SPH will behave in an approximately correct manner, giving zero pressure for fluids and zero surface stresses for solids, because of the deficiency of particles at the boundary. This is the reason why conventional SPH gives physically reasonable results at free surfaces. Contact between bodies occurs by smoothing over all particles, regardless of material. Although simple this approach gives physically incorrect results. 
Campbell [11] made an early attempt to introduce a more systematic treatment of boundary condition by re-considering the original kernel integral estimates and taking into account the boundary conditions through residual terms in the integral by parts. Probably the most sophisticated work on boundary conditions in SPH is due to Takeda et al. [46], who have applied SPH to a variety of viscous flows. A similar approach has also been used to a limited extent by Libersky [26, 27] with the ghost particles added to accomplish a reflected symmetrical surface boundary condition. In, Belytschko, $\mathrm{Lu}$ and $\mathrm{Gu}[6]$ the essential boundary conditions were imposed by the use of Lagrange multipliers leading to an awkward structure of the linear algebraic equations, which are not positive definite. Krongauz and Belytschko [22] proposed a simpler technique for the treatment of the essential boundary conditions in meshless methods, by employing a string of finite elements along the essential boundaries. This allowed for the boundary conditions to be treated accurately, but reintroduced the shortcomings inherent to structured meshes.

Randles et al. [41, 42] were first to propose a more general treatment of boundary conditions based on an extension of the ghost particle method. In this, the boundary is considered to be a surface one half of the local smoothing length away from the so-called boundary particles. A boundary condition is applied to a field variable by assigning the same boundary value of the variable to all ghost particles. A constraint is imposed on the boundary by interpolating it smoothly between the specified boundary particle value and the calculated values on the interior particles. This serves to communicate to the interior particles the effect of the specific boundary condition. There are two main difficulties in this:

- Definition of the boundary (surface normal at the vertices).

- Communication of the boundary value of a dependent variable from the boundary to internal particles.

A penalty contact algorithm for SPH was developed at Cranfield by Campbell and Vignjevic [51]. This algorithm was tested on normalised SPH using the Randles approach for free surfaces. The contact algorithm considered only particle-particle interactions, and allowed contact and separation to be correctly simulated. However tests showed that this approach often excited zero-energy modes.

Another unconventional solution to the SPH tensile instability problem was first proposed by Dyka [13] in which the stresses are calculated at the locations other than the SPH particles. The results achieved in $1 \mathrm{D}$ were encouraging but a rigorous stability analysis was not performed. A 2D version of this approach was investigated by Vignjevic [51], based on the normalised version of SPH. This investigation showed that extension to $2 \mathrm{D}$ was possible, although general boundary condition treatment and simulation of large deformations would require further research.

To utilise the best aspects of the FE and SPH methods it was necessary to develop interfaces for the linking of SPH nodes with standard finite element grids $[18,19]$ 
and contact algorithms for treatment of contact between the two particles and elements [54].

From the review of the development of meshless methods, given above, the following major problems can be identified: consistency, stability and the treatment of boundary conditions.

\section{Basic formulation}

The spatial discretisation of the state variables is provided by a set of points. Instead of a grid, SPH uses a kernel interpolation to approximate the field variables at any point in a domain. For instance, an estimate of the value of a function $f(x)$ at the location $x$ is given in a continuous form by an integral of the product of the function and a kernel (weighting) function $W\left(x-x^{\prime}, h\right)$ :

$$
\langle f(x)\rangle=\int f\left(x^{\prime}\right) W\left(x-x^{\prime}, h\right) d x^{\prime}
$$

Where: the angle brackets \langle\rangle denote a kernel approximation, $h$ is a parameter that defines size of the kernel support known as the smoothing length, and $x^{\prime}$ is new independent variable.

The kernel function usually has the following properties:

Compact support, which means that it's zero everywhere but on a finite domain inside the range of the smoothing length $2 \mathrm{~h}$ :

$W\left(x-x^{\prime}, h\right)=0$ for $\left|x-x^{\prime}\right| \geq 2 h$

Normalised

$\int W\left(x-x^{\prime}, h\right) d x^{\prime}=1$

These requirements, formulated by Lucy [28], ensure that the kernel function reduces to the Dirac delta function when $h$ tends to zero:

$\lim _{h \rightarrow 0} W\left(x-x^{\prime}, h\right)=\delta\left(x-x^{\prime}, h\right)$

And therefore, it follows that:

$\lim _{h \rightarrow 0}\langle f(x)\rangle=f(x)$

If the function $f(x)$ is only known at $N$ discrete points, the integral of equation 1 can be approximated by a summation:

$\langle f(x)\rangle=\sum_{j=1}^{N} \frac{m^{j}}{\rho^{j}} f\left(x^{j}\right) W\left(x-x^{j}, h\right)$

where $\frac{m^{j}}{\rho^{j}}$ is the volume associated to the point or particle $j$. In SPH literature, the term particles is misleading as in fact these particles have to be thought of as interpolation points rather than mass elements. 
Equation 6 constitutes the basis of SPH method. The value of a variable at a particle, denoted by superscript $i$, is calculated by summing the contributions from a set of neighbouring particles (Figure 1), denoted by superscript $j$ and for which the kernel function is not zero:

$$
\left\langle f\left(x^{i}\right)\right\rangle=\sum_{j=1}^{N} \frac{m^{j}}{\rho^{j}} f\left(x^{j}\right) W\left(x^{i}-x^{j}, h\right)
$$

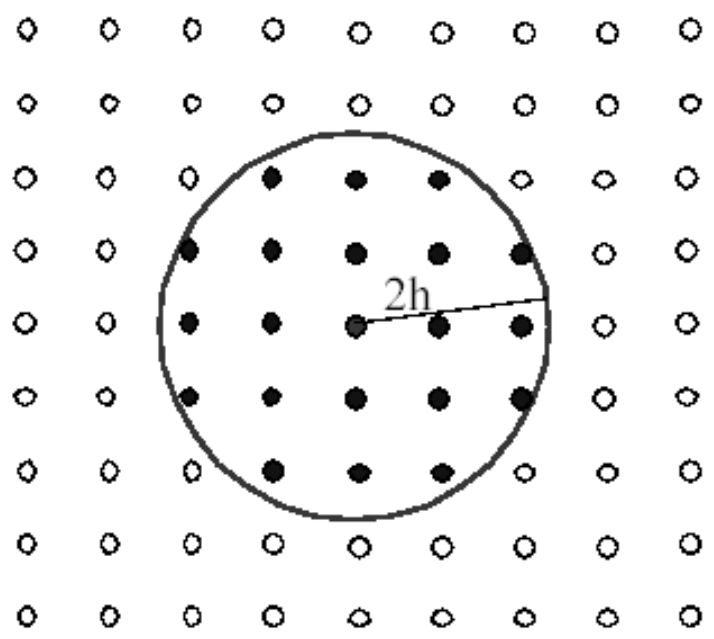

Figure 1: Set of neighbouring particles

\section{Conservation equations}

The conservation equations in Lagrangian framework are given by:

$$
\begin{aligned}
& \frac{d \rho}{d t}=-\rho \frac{\partial v_{\alpha}}{\partial x_{\alpha}} \\
& \frac{d v_{\alpha}}{d t}=\frac{1}{\rho} \frac{\partial \sigma_{\alpha \beta}}{\partial x_{\beta}} \text { or } \frac{d v_{\alpha}}{d t}=\frac{\partial}{\partial x_{\beta}}\left(\frac{\sigma_{\alpha \beta}}{\rho}\right)+\frac{\sigma_{\alpha \beta}}{\rho^{2}} \frac{\partial \rho}{\partial x_{\beta}} \\
& \frac{d E}{d t}=\frac{\sigma_{\alpha \beta}}{\rho} \frac{\partial v_{\alpha}}{\partial x_{\beta}} \text { or } \frac{d E}{d t}=\frac{\sigma_{\alpha \beta}}{\rho^{2}} \frac{\partial\left(\rho v_{\alpha}\right)}{\partial x_{\beta}}-\frac{\sigma_{\alpha \beta} v_{\alpha}}{\rho^{2}} \frac{\partial \rho}{\partial x_{\beta}} \\
& \text { with } v_{\alpha}=\frac{d x_{\alpha}}{d t}
\end{aligned}
$$

The subscripts $\alpha$ and $\beta$ denote the component.

Equations $9 \mathrm{~b}$ and $10 \mathrm{~b}$ are the forms proposed by Monaghan [32]. The kernel interpolation allows the derivation of the basic SPH form of these conservation equations as:

$\left\langle\frac{d \rho}{d t}\right\rangle=-\int W \rho^{\prime} \frac{\partial v_{\alpha}^{\prime}}{\partial x_{\alpha}^{\prime}} d x^{\prime}$ 


$$
\begin{aligned}
\left\langle\frac{d v_{\alpha}}{d t}\right\rangle & =\int W \frac{\partial}{\partial x_{\beta}^{\prime}}\left(\frac{\sigma_{\alpha \beta}^{\prime}}{\rho^{\prime}}\right) d x^{\prime}+\int W \frac{\sigma_{\alpha \beta}^{\prime}}{\rho^{\prime 2}} \frac{\partial \rho^{\prime}}{\partial x^{\prime}{ }_{\beta}} d x^{\prime} \\
\left\langle\frac{d E}{d t}\right\rangle & =\int W \frac{\sigma_{\alpha \beta}^{\prime}}{\rho^{\prime 2}} \frac{\partial\left(\rho^{\prime} v_{\alpha}^{\prime}\right)}{\partial x^{\prime}{ }_{\beta}} d x^{\prime}-\int W \frac{\sigma_{\alpha \beta}^{\prime} v_{\alpha}^{\prime}}{\rho^{\prime 2}} \frac{\partial \rho^{\prime}}{\partial x_{\beta}^{\prime}} d x^{\prime}
\end{aligned}
$$

All the equations above contain integrals of the form:

$$
\int W f\left(x^{\prime}\right) \frac{\partial g\left(x^{\prime}\right)}{\partial x^{\prime}} d x^{\prime}
$$

Using a development in a Taylor series about $x^{\prime}=x$, it follows:

$\int W f\left(x^{\prime}\right) \frac{\partial g\left(x^{\prime}\right)}{\partial x^{\prime}} d x^{\prime}=\int\left\{f(x) \frac{\partial g(x)}{\partial x}+\left(x-x^{\prime}\right) \frac{d}{d x}\left(f(x) \frac{\partial g(x)}{\partial x}\right)+\ldots\right\} W d x^{\prime}$

As $W$ is an even function, the terms containing odd powers of $x-x^{\prime}$ vanish. Neglecting second and higher order terms, which is consistent with the overall order of the method, gives:

$\int W f\left(x^{\prime}\right) \frac{\partial g\left(x^{\prime}\right)}{\partial x^{\prime}} d x^{\prime}=\left(f\left(x^{\prime}\right) \frac{\partial g\left(x^{\prime}\right)}{\partial x^{\prime}}\right)_{x^{\prime}=x}$

Substituting $\left\langle\frac{\partial g(x)}{\partial x}\right\rangle$ for $\frac{\partial g(x)}{\partial x}$ gives:

$$
\left(f\left(x^{\prime}\right) \frac{\partial g\left(x^{\prime}\right)}{\partial x^{\prime}}\right)_{x^{\prime}=x}=f(x) \int W \frac{\partial g\left(x^{\prime}\right)}{\partial x^{\prime}} d x^{\prime}
$$

Using the last relation in equations 11,12 and 13 gives

$$
\begin{aligned}
\left\langle\frac{d \rho}{d t}\right\rangle & =-\rho \int W \frac{\partial v_{\alpha}^{\prime}}{\partial x_{\alpha}^{\prime}} d x^{\prime} \\
\left\langle\frac{d v_{\alpha}}{d t}\right\rangle & =\int W \frac{\partial}{\partial x_{\beta}^{\prime}}\left(\frac{\sigma_{\alpha \beta}^{\prime}}{\rho^{\prime}}\right) d x^{\prime}+\frac{\sigma_{\alpha \beta}}{\rho^{2}} \int W \frac{\partial \rho^{\prime}}{\partial x_{\beta}^{\prime}} d x^{\prime} \\
\left\langle\frac{d E}{d t}\right\rangle & =\frac{\sigma_{\alpha \beta}}{\rho^{2}} \int W \frac{\partial\left(\rho^{\prime} v_{\alpha}^{\prime}\right)}{\partial x_{\beta}^{\prime}} d x^{\prime}-\frac{\sigma_{\alpha \beta} v_{\alpha}}{\rho^{2}} \int W{\frac{\partial \rho^{\prime}}{\partial x_{\beta}^{\prime}}}_{\beta} d x^{\prime}
\end{aligned}
$$

All equations include kernel approximations of spatial derivatives:

$$
\left\langle\frac{\partial f(x)}{\partial x_{\alpha}}\right\rangle=\int W \frac{\partial f\left(x^{\prime}\right)}{\partial x_{\alpha}^{\prime}} d x^{\prime}
$$

Integrating by part gives:

$$
\left\langle\frac{\partial f(x)}{\partial x_{\alpha}}\right\rangle=W f(x)-\int f(x) \frac{\partial W}{\partial x_{\alpha}^{\prime}} d x^{\prime}
$$


The first term of the second member can be rewritten:

$W f(x)=\int \frac{\partial\left(W f\left(x^{\prime}\right)\right)}{\partial x^{\prime}} d x^{\prime}$

Using Green's theorem, it follows:

$\int \frac{\partial\left(W f\left(x^{\prime}\right)\right)}{\partial x^{\prime}} d x^{\prime}=\int_{S} W f\left(x^{\prime}\right) n_{i} d S$

The surface integral is zero if the domain of integration is larger than the compact support of $W$ or if the field variable assumes zero value on the boundary of the body (free surface). If none of these conditions is satisfied, modifications should be made to account for boundary conditions.

One should note that in equations 18,19 and 20 the spatial derivatives of the field variables are substituted by the derivatives of the kernel:

$\int W \frac{\partial f\left(x^{\prime}\right)}{\partial x_{\alpha}^{\prime}} d x^{\prime}=-\int f(x) \frac{\partial W}{\partial x_{\alpha}^{\prime}} d x^{\prime}$

It follows:

$$
\begin{aligned}
\left\langle\frac{d \rho}{d t}\right\rangle & =\rho \int v_{\alpha}^{\prime} \frac{\partial W}{\partial x_{\alpha}^{\prime}} d x^{\prime} \\
\left\langle\frac{d v_{\alpha}}{d t}\right\rangle & =-\int \frac{\sigma_{\alpha \beta}^{\prime}}{\rho^{\prime}} \frac{\partial W}{\partial x_{\beta}^{\prime}} d x^{\prime}-\frac{\sigma_{\alpha \beta}}{\rho^{2}} \int \rho^{\prime} \frac{\partial W}{\partial x_{\beta}^{\prime}} d x^{\prime} \\
\left\langle\frac{d E}{d t}\right\rangle & =-\frac{\sigma_{\alpha \beta}}{\rho^{2}} \int \rho^{\prime} v_{\alpha}^{\prime} \frac{\partial W}{\partial x_{\beta}^{\prime}} d x^{\prime}+\frac{\sigma_{\alpha \beta} v_{\alpha}}{\rho^{2}} \int \rho^{\prime} \frac{\partial W}{\partial x_{\beta}^{\prime}} d x^{\prime}
\end{aligned}
$$

The final step is to convert the continuous volume integrals to sums over discrete interpolation points. Finally, after a few arrangements in order to improve the consistency between all equations, the most common form of the SPH discretised conservation equations are obtained:

$$
\begin{aligned}
& \frac{d \rho^{i}}{d t}=\rho^{i} \sum_{j=1}^{N} \frac{m^{j}}{\rho^{j}}\left(v_{\beta}^{j}-v_{\beta}^{i}\right) \frac{\partial W^{i j}}{\partial x_{\beta}^{i}} \\
& \frac{d v_{\alpha}^{i}}{d t}=-\sum_{j=1}^{N} m^{j}\left(\frac{\sigma_{\alpha \beta}^{j}}{\rho^{j 2}}-\frac{\sigma_{\alpha \beta}^{i}}{\rho^{i 2}}\right) \frac{\partial W^{i j}}{\partial x_{\beta}^{i}} \\
& \frac{d E^{i}}{d t}=-\frac{\sigma_{\alpha \beta}^{i}}{\rho^{i 2}} \sum_{j=1}^{N} m^{j}\left(v_{\alpha}^{j}-v_{\alpha}^{i}\right) \frac{\partial W^{i j}}{\partial x_{\beta}^{i}}
\end{aligned}
$$

where $W^{i j}=W\left(x^{i}-x^{j}, h\right)$ 


\section{Kernel function}

To complete the discretisation one has to define the kernel function. Numerous possibilities exist. A large number of kernel function types are discussed in literature, ranging from polynomial to Gaussian. The most common is the B-spline kernel that was proposed by Monaghan [32]:

$$
W(v, h)=\frac{C}{h^{D}} \begin{cases}\left(1-\frac{3}{2} v^{2}+\frac{3}{4} v^{3}\right) & v<1 \\ \frac{1}{4}(2-v)^{3} & 1 \leq v \leq 2 \\ 0 & \text { otherwise }\end{cases}
$$

where

$v=\frac{\left|x-x^{\prime}\right|}{h}$,

$D$ is the number of dimensions of the problem (i.e. 1, 2 or 3),

$C$ is the scaling factor which depends on the number of dimensions and ensures that the consistency conditions 2 and 3 are satisfied:

$$
C= \begin{cases}\frac{2}{3} & D=1 \\ \frac{10}{7 \pi} & D=2 \\ \frac{1}{\pi} & D=3\end{cases}
$$

\section{Variable smoothing length}

If large deformations occur, particles can largely separate from each other. If the smoothing length remains constant, the particle spacing can become so large than particles will no more interact. On the other hand, in compression, a lot of particles might enter in the neighbouring of each other, which can significantly slow down the calculation. In order to avoid these problems, Benz [8] proposed the use of a variable smoothing length. The intent was to maintain a healthy neighbourhood as continuum deforms. The equation for evolution of $h$ derived by [8] is:

$$
h=h_{0}\left(\frac{\rho_{0}}{\rho}\right)^{\frac{1}{n}}
$$

where $h_{0}$ and $\rho_{0}$ are initial smoothing length and density and $n$ is the number of dimensions of the problem. 
Another frequently used equation of evolution based on conservation of mass is:

$$
\frac{d h}{d t}=\frac{1}{n} h \operatorname{div}(v)
$$

where $\operatorname{div}(v)$ is the divergence of velocity.

\section{Neighbour search}

An important step in the SPH computation is the neighbour search. This task can be extremely time consuming. The neighbour search routine lists the particles that are inside the neighbourhood of each particle at each time step. A direct search between every particle is particularly inefficient. A bucket sort algorithm is more efficient. In this method, an underlying grid of side $2 h$ is generated and the particles are sorted according to the box in which they are located (Figure 2). Then for each particle, the neighbours are searched among the particles contained in the same box and the surrounding boxes. This allows the computational time to be cut down from a default time proportional to $N^{2}$ for a direct search to $N \log N$, where $N$ is the total number of particles.

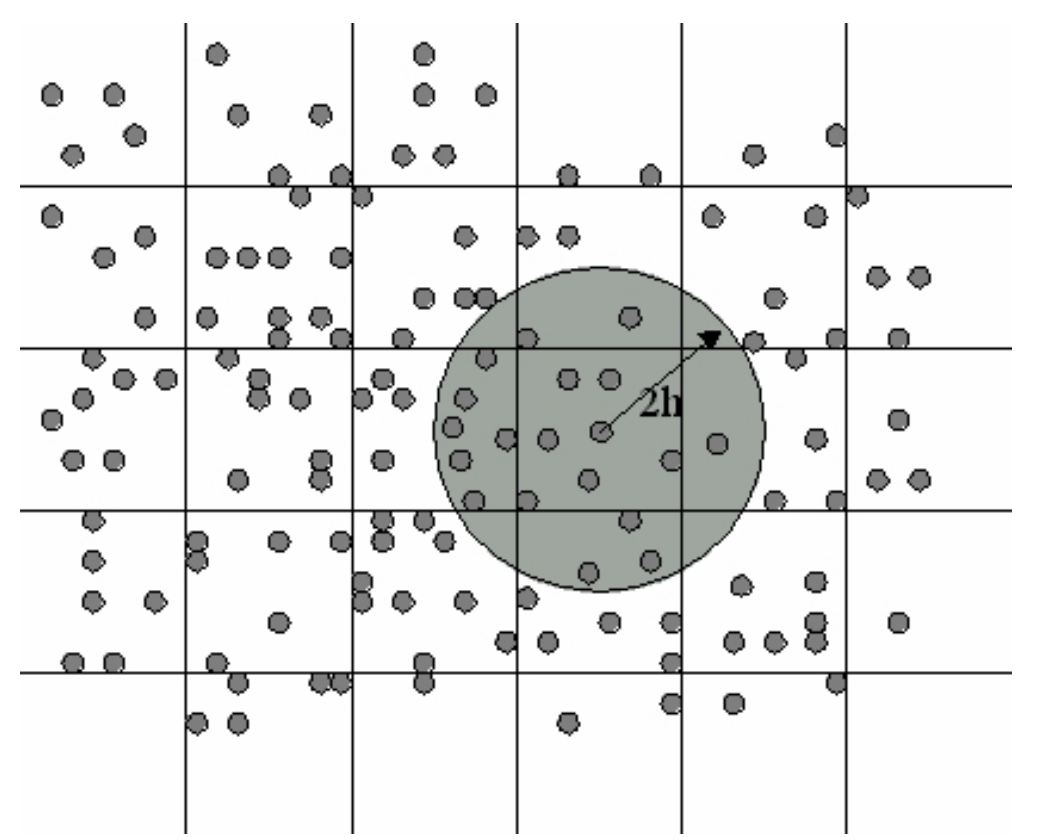

Figure 2: Bucket sort and neighbour search

\section{SPH shortcomings}

The basic SPH method has shown several problems when used to model a solid body:

- Consistency

- Tensile instability

- Zero-energy modes 


\section{Consistency}

The SPH method in its continuous form is inconsistent within $2 \mathrm{~h}$ of the domain boundaries due to the kernel support incompleteness. In its discrete form the method loses its $0^{\text {th }}$ order consistency not only in the vicinity of boundaries but also over the rest of the domain if particles have an irregular distribution. Meglicki [29] showed that node disorder results in a systematic error. Therefore, a proper SPH grid should be as regular as possible and not contain large discrepancies in order to perform most accurate simulation.

The first order consistency of the method can be achieved in two ways. Firstly, by correcting the kernel function, second, by correcting the discrete form of the convolution integral of the SPH interpolation. Johnson [21] uses this correction procedure and proposed the Normalised Smoothing Function. Vignjevic [51] also implemented a kernel normalisation and correction to lead to a Corrected Normalised Smooth Particle Hydrodynamics (CNSPH) method which is first order consistent. The full derivation of this correction is given below. In SPH methods based on a corrected kernel, it is no-longer possible to ignore boundary conditions. In basic SPH, free surface boundary conditions are not imposed and are simply ignored as variables tends to zero at boundaries because of the deficiency of neighbour particles.

\section{Derivation of normalised corrected gradient SPH formula}

The approximation of fields using a Normalised Corrected SPH (NCSPH) interpolation has been published [42, 51, 9]. Some authors have chosen to use properties of the integrals of motion (linear and angular momentum) to derive Normalisation and Gradient Correction for kernel interpolation, see [9]. This approach lacks generality and does not provide the insight into the origin and the nature of the problem. A full derivation of the correction proposed by Vignjevic [51], which has not been published before, is given below. The derivation is based on the homogeneity and isotropy of space, the space properties, which have as a consequence conservation of linear and angular momentum, see [24]. The mixed correction insures that homogeneity and isotropy of space are preserved in the process of spatial discretisation.

An interpolation technique should not affect homogeneity of space. One way of demonstrating this is to prove that the interpolation of the solution space itself is independent of a translation of the coordinate axes. In order to express this statement mathematically one can start by writing the general expression for the SPH interpolation of a vector field:

$$
\left.\langle\overrightarrow{\mathbf{F}}(\overrightarrow{\mathbf{x}})\rangle\right|_{\overrightarrow{\mathbf{x}}=\overrightarrow{\mathbf{x}}_{\mathrm{i}}}=\sum_{\mathrm{j}} \frac{\mathrm{m}_{\mathrm{j}}}{\rho_{\mathrm{j}}} \overrightarrow{\mathbf{F}}\left(\overrightarrow{\mathbf{x}}_{\mathrm{j}}\right) \mathrm{W}\left(\overrightarrow{\mathbf{x}}_{\mathrm{i}}-\overrightarrow{\mathbf{x}}_{\mathrm{j}}\right)
$$


If the field to be interpolated is the solution space then $\overrightarrow{\mathbf{F}}=\overrightarrow{\mathbf{x}}$ and equation 36 becomes:

$$
\left.\langle\overrightarrow{\mathbf{x}}\rangle\right|_{\overrightarrow{\mathbf{x}}=\overrightarrow{\mathbf{x}}_{\mathrm{i}}}=\sum_{\mathrm{j}} \frac{\mathrm{m}_{\mathrm{j}}}{\rho_{\mathrm{j}}} \overrightarrow{\mathbf{x}}_{\mathrm{j}} \mathrm{W}\left(\overrightarrow{\mathbf{x}}_{\mathrm{i}}-\overrightarrow{\mathbf{x}}_{\mathrm{j}}\right)
$$

In a different, translated coordinate system, this equation is:

$$
\left.\left\langle\overrightarrow{\mathbf{x}}^{\prime}\right\rangle\right|_{\overrightarrow{\mathbf{x}}^{\prime}=\overrightarrow{\mathbf{x}}_{\mathrm{i}}^{\prime}}=\sum_{\mathrm{j}} \frac{\mathrm{m}_{\mathrm{j}}}{\rho_{\mathrm{j}}} \overrightarrow{\mathbf{x}}_{\mathrm{j}}^{\prime} \mathrm{W}\left(\overrightarrow{\mathbf{x}}_{\mathrm{i}}^{\prime}-\overrightarrow{\mathbf{x}}_{\mathrm{j}}^{\prime}\right)
$$

Where $\overrightarrow{\mathbf{x}}^{\prime}$ is the coordinate vector in the new coordinate system. If the translation vector by which the origin of the coordinate system was moved is defined as $\Delta \overrightarrow{\mathbf{x}}$ then the relationship between $\overrightarrow{\mathbf{x}}$ and $\overrightarrow{\mathbf{x}}^{\prime}$ is:

$$
\overrightarrow{\mathbf{x}}^{\prime}=\overrightarrow{\mathbf{x}}-\Delta \overrightarrow{\mathbf{x}}
$$

If the interpolated coordinates of a point are independent of the translation of coordinate axes then the following should hold:

$$
\left\langle\overrightarrow{\mathbf{x}}^{\prime}\right\rangle=\langle\overrightarrow{\mathbf{x}}\rangle-\Delta \overrightarrow{\mathbf{x}}
$$

By substituting equation 40 into equation 39 for both $\overrightarrow{\mathbf{x}}_{\mathrm{i}}$ and $\overrightarrow{\mathbf{x}}_{\mathrm{j}}$ one obtains:

$$
\left\langle\overrightarrow{\mathbf{x}}^{\prime}\right\rangle=\sum_{j} \frac{\mathrm{m}_{\mathrm{j}}}{\rho_{\mathrm{j}}} \overrightarrow{\mathbf{x}}_{\mathrm{j}} \mathrm{W}\left(\overrightarrow{\mathbf{x}}_{\mathrm{i}}-\overrightarrow{\mathbf{x}}_{\mathrm{j}}\right)-\sum_{\mathrm{j}} \frac{\mathrm{m}_{\mathrm{j}}}{\rho_{\mathrm{j}}} \Delta \overrightarrow{\mathbf{x}} \mathrm{W}\left(\overrightarrow{\mathbf{x}}_{\mathrm{i}}-\overrightarrow{\mathbf{x}}_{\mathrm{j}}\right)
$$

or

$$
\left\langle\overrightarrow{\mathbf{x}}^{\prime}\right\rangle=\langle\overrightarrow{\mathbf{x}}\rangle-\Delta \overrightarrow{\mathbf{x}} \sum_{\mathrm{j}} \frac{\mathrm{m}_{\mathrm{j}}}{\rho_{\mathrm{j}}} \mathrm{W}\left(\overrightarrow{\mathbf{x}}_{\mathrm{i}}-\overrightarrow{\mathbf{x}}_{\mathrm{j}}\right)
$$

By comparison of equation 42 and equation 40 it is clear that the discretised space will only be homogeneous if the following condition is satisfied:

$$
\sum_{j} \frac{m_{j}}{\rho_{j}} W\left(\overrightarrow{\mathbf{x}}_{i}-\overrightarrow{\mathbf{x}}_{j}\right)=1
$$

Similarly, an interpolation technique should not affect isotropy of space. One way of demonstrating this is to prove that the interpolation of the solution space itself is independent of a rotation of the coordinate axes. The same holds for the SPH approximation. The change in coordinates due to a rotation of the coordinate axes is:

$$
\overrightarrow{\mathbf{x}}^{\prime}=\mathbf{C} \overrightarrow{\mathbf{x}}
$$

where $\mathbf{C}$ is the rotation matrix. For small rotations this can also be written as: 
$\overrightarrow{\mathbf{x}}^{\prime}=\overrightarrow{\mathbf{x}}-\Delta \vec{\phi} \times \overrightarrow{\mathbf{x}}$

where $\Delta \vec{\phi}$ is the rotation vector.

If one wants to ensure that the SPH approximation does maintain the fact that space is isotropic then the approximation has to satisfy the following condition:

$\left\langle\overrightarrow{\mathbf{x}}^{\prime}\right\rangle \equiv\langle\mathbf{C} \overrightarrow{\mathbf{x}}\rangle=\mathbf{C}\langle\overrightarrow{\mathbf{x}}\rangle$

or

$\langle\mathbf{C}\rangle=\mathbf{C}$

This means that the rotation matrix has to be approximated exactly.

In order to develop this equation one can start by rewriting

$$
\begin{aligned}
\overrightarrow{\mathbf{x}}^{\prime} & =\overrightarrow{\mathbf{x}}-\Delta \vec{\phi} \times \overrightarrow{\mathbf{x}} \\
& =\overrightarrow{\mathbf{x}}-\nabla(\Delta \vec{\phi} \times \overrightarrow{\mathbf{x}}) \cdot \overrightarrow{\mathbf{x}} \\
& =\overrightarrow{\mathbf{x}}-\phi^{\mathrm{x}} \overrightarrow{\mathbf{x}} \\
& =\left(\mathbf{I}-\phi^{\mathbf{x}}\right) \overrightarrow{\mathbf{x}}
\end{aligned}
$$

where $\Delta \phi^{\mathrm{x}}$ is a skew-symmetric matrix:

$$
\Delta \phi^{\mathrm{x}}=\left[\begin{array}{ccc}
0 & -\Delta \phi_{\mathrm{z}} & \Delta \phi_{\mathrm{y}} \\
\Delta \phi_{\mathrm{z}} & 0 & -\Delta \phi_{\mathrm{x}} \\
-\Delta \phi_{\mathrm{y}} & \Delta \phi_{\mathrm{x}} & 0
\end{array}\right]
$$

This means that, for small rotations, the rotation matrix is given by:

$\mathbf{C}=\mathbf{I}-\phi^{\mathbf{x}}$

The approximation of the rotated coordinates is:

$\left\langle\overrightarrow{\mathbf{x}}^{\prime}\right\rangle \equiv\langle\mathbf{C} \overrightarrow{\mathbf{x}}\rangle=\langle\mathbf{C}\rangle\langle\overrightarrow{\mathbf{x}}\rangle=\left\langle\mathbf{I}-\phi^{\mathrm{x}}\right\rangle\langle\overrightarrow{\mathbf{x}}\rangle$

This means that the requirement on the interpolation is:

$$
\mathbf{I}-\phi^{\mathrm{x}}=\left\langle\mathbf{I}-\phi^{\mathrm{x}}\right\rangle
$$

or

$\phi^{\mathrm{x}}=\left\langle\phi^{\mathrm{x}}\right\rangle$

Expanding this expression leads to: 


$$
\begin{aligned}
\left\langle\phi^{\mathrm{x}}\right\rangle & =\sum_{\mathrm{j}} \frac{\mathrm{m}_{\mathrm{j}}}{\rho_{\mathrm{j}}} \Delta \vec{\phi} \times \overrightarrow{\mathrm{x}}_{\mathrm{j}} \nabla \mathrm{W}\left(\overrightarrow{\mathrm{x}}_{\mathrm{i}}-\overrightarrow{\mathrm{x}}_{\mathrm{j}}\right) \\
& =\sum_{\mathrm{j}} \frac{\mathrm{m}_{\mathrm{j}}}{\rho_{\mathrm{j}}}\left(\phi^{\mathrm{x}} \overrightarrow{\mathrm{x}}_{\mathrm{j}}\right) \nabla \mathrm{W}\left(\overrightarrow{\mathrm{x}}_{\mathrm{i}}-\overrightarrow{\mathrm{x}}_{\mathrm{j}}\right) \\
& =\phi^{\mathrm{x}} \sum_{\mathrm{j}} \frac{\mathrm{m}_{\mathrm{j}}}{\rho_{\mathrm{j}}} \overrightarrow{\mathrm{x}}_{\mathrm{j}} \nabla \mathrm{W}\left(\overrightarrow{\mathrm{x}}_{\mathrm{i}}-\overrightarrow{\mathrm{x}}_{\mathrm{j}}\right)
\end{aligned}
$$

Therefore to preserve space isotropy, i.e. $\phi^{\mathrm{x}}=\left\langle\phi^{\mathrm{x}}\right\rangle$ the following condition has to be satisfied.

$$
\sum_{j} \frac{m_{j}}{\rho_{j}} \vec{x}_{j} \nabla W\left(\vec{x}_{i}-\vec{x}_{j}\right)=\mathbf{I}
$$

The form of the normalised kernel function and the approximation of the first order derivatives which provides first order consistency is given in Table 1 below.

\begin{tabular}{|l|l|l|}
\hline & Space Homogeneity & Space Anisotropy \\
\hline Condit. & $\sum_{j=1}^{n n b r} \frac{m_{j}}{\rho_{j}} W\left(x_{i}-x_{j}, h\right)=1$ & $\sum_{j=1}^{n n b r} \frac{m_{j}}{\rho_{j}} \mathbf{x}_{j} \otimes \nabla W\left(x_{i}-x_{j}, h\right)=\mathbf{1}$ \\
\hline $\begin{array}{l}\text { Normalised } \\
\text { - Corrected }\end{array}$ & $\widetilde{W}_{i j}=\frac{W\left(x_{i}-x_{j}, h\right)}{\sum_{j=1}^{n n b r} \frac{m_{j}}{\rho_{j}} W\left(x_{i}-x_{j}, h\right)}$ & $\widetilde{\nabla} \widetilde{\mathbf{w}}{ }_{i j}=\nabla \widetilde{\mathbf{w}}_{i j}\left(\sum_{j=1}^{n n b r} \frac{m_{j}}{\rho_{j}} x_{j} \otimes \nabla \widetilde{\mathbf{w}}_{i j}\right)^{-1}$ \\
\hline
\end{tabular}

Table 1: Corrected forms of the kernel function and its gradient

Using the NCSPH approximations the conservation equations assume the following form:

$$
\begin{aligned}
& \dot{\rho}_{i}=\rho_{i} \sum_{j=1}^{n n b r} \frac{m_{j}}{\rho_{j}}\left(\overrightarrow{\mathbf{v}}_{j}-\overrightarrow{\mathbf{v}}_{i}\right) \cdot \widetilde{\nabla} \widetilde{\mathbf{W}}_{i j} \\
& \dot{\overrightarrow{\mathbf{v}}}_{\mathbf{i}}=\sum_{j=1}^{n n b r} m_{j}\left(\frac{\boldsymbol{\sigma}_{i}}{\rho_{i}{ }^{2}}+\frac{\boldsymbol{\sigma}_{j}}{\rho_{j}{ }^{2}}\right) \cdot \widetilde{\nabla} \widetilde{\mathbf{W}}_{i j} \\
& \dot{e}=-\frac{\boldsymbol{\sigma}_{i}}{\rho_{i}^{2}} \sum_{j=1}^{n n b r} m_{j}\left(\overrightarrow{\mathbf{v}}_{j}-\overrightarrow{\mathbf{v}}_{i}\right) \cdot \widetilde{\nabla} \widetilde{\mathbf{W}}_{i j}
\end{aligned}
$$




\section{Tensile instability}

A Von Neumann stability analysis of the SPH method was conducted Swegle [45] and Balsara [59] separately. This has revealed that the SPH method suffers from a tensile instability. This instability manifests itself as a clustering of the particles, which resembles fracture and fragmentation, but is in fact a numerical artefact. Swegle [45] concluded that the instability doesn't result from the numerical time integration algorithm, but rather from an effective stress resulting from a nonphysical negative modulus being produced by the interaction between the constitutive relation and the kernel interpolation. In other words the kernel interpolation used in spatial discretisation changes the nature of original partial differential equations. These changes in the effective stress amplify, rather than reduce, perturbations in the strain. From Swegle's stability analysis it emerged that the criterion for stability was that:

$$
W^{\prime \prime} \sigma>0
$$

where $W^{\prime \prime}$ is the second derivative of $W$ with respect to its argument and $\sigma$ is the stress, negative in compression and positive in tension.

This criterion states that instability can also occur in compression, not only in tension. Indeed, if the slope of the derivative of the kernel function is positive, the method is unstable in tension and stable in compression and if the slope is negative, it is unstable in compression and stable in tension.

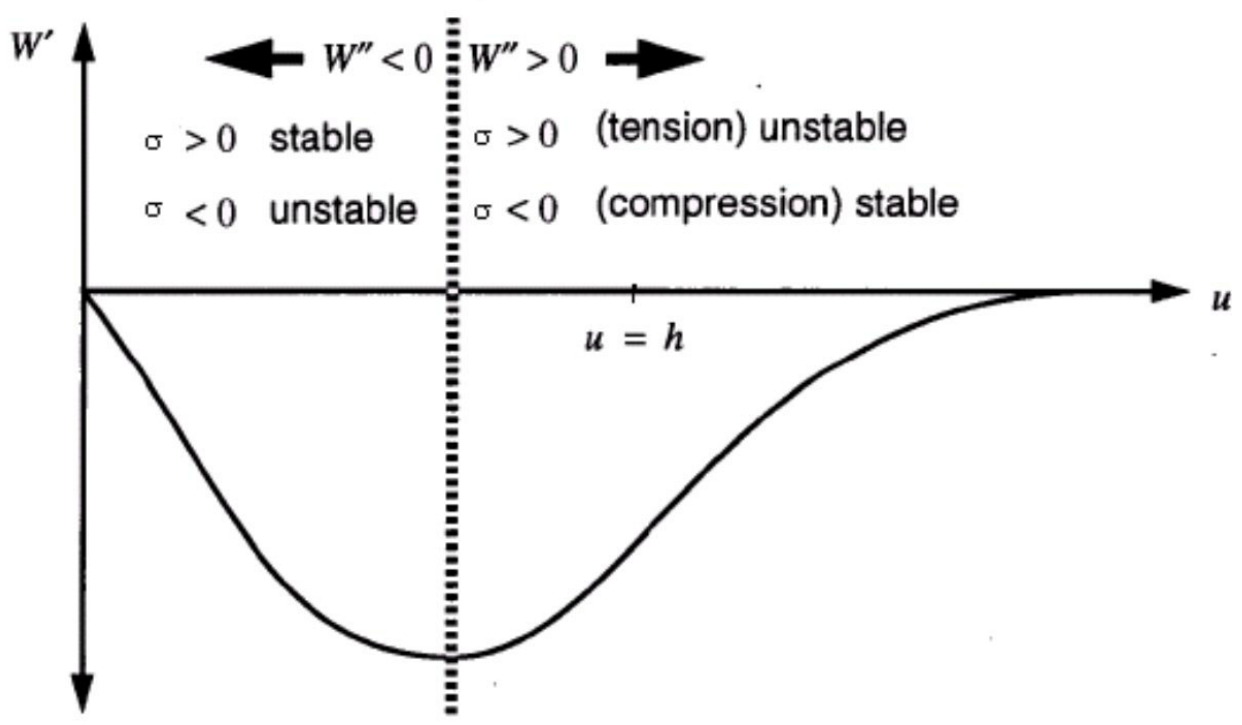

Figure 3: Stability regimes for the B-spline kernel function [45]

The fact that this instability manifests itself most often in tension can be explained. Figure 3 shows the stability regime for the B-spline kernel function. The minimum of the derivative is situated at $u=2 / 3 h$. In standard configurations, the particle spacing is equal to the smoothing length, $u=h$. Thus, standard configurations are unstable in tension. This explains why this unstable phenomenon is generally observed in tension and hence, its misleading name "tensile instability". 
In order to remedy this problem several solutions have been proposed. Guenther [15] and Wen [55] have proposed a solution, known as Conservative Smoothing. Randles and Libersky [41] proposed adding dissipative terms, which is related to conservative smoothing. Dyka [13] proposed an original solution by using a non-collocated discretisation of stress and velocity points. At one set of points the stresses are evaluated, while the momentum equation is calculated at another set of points. The 'stress' points are equivalent to the Gauss quadrature points in FE, the other set of points is equivalent to the element nodes. This approach was extended to two dimensions, in combination with kernel normalisation, by Vignjevic and Campbell [51]. Other solutions were proposed, for instance see [37]. The former proposes a corrective SPH method by enforcing higher order consistency, while the latter proposes the addition of an artificial force to stabilise the computation. Recently Randles and Libersky combined MLS interpolation with the stress and velocity point approach. They called this approach the Dual Particle Dynamics method [58].

The conservative smoothing and the artificial repulsive forces methods have limited applicability and have to be used with caution because they affect the strength of material being modelled. At present, the most promising approach is noncollocational spatial discretisation. This problem is in the focus of attention of a number of researchers working on mesh-less methods.

\section{Zero-energy modes}

Zero-energy modes are a problem that is not unique to particle methods. These spurious modes, which correspond to modes of deformation characterised by a pattern of nodal displacement that produces zero strain energy, can also be found in the finite difference and finite element methods.

Swegle [45] was first to showed that SPH suffers from zero energy modes. These modes arise from the nodal under integration. The fundamental cause is that all field variables and their derivatives are calculated at the same locations (particle positions). For instance, for an oscillatory velocity field, illustrated in Figure 1, the kernel approximation would give negligible gradients and consequently stresses at the particles. These modes of deformation are not resisted and can be easily exited by rapid impulsive loading. Another explanation can be found in the origin of the kernel approximation. As the kernel approximation, which is the basis of SPH, is an interpolation of a set of discrete data, a constant field, can be fit with a sinusoidal curve/surface if the order of the interpolation is high enough.

Figure 4 illustrates this spurious mode for a field in 1D SPH. If one would approximate the derivative of the field shown in Figure 4 with a central difference formula: 


$$
\left.\frac{d f}{d x}\right|_{x=x_{i}}=\frac{f\left(x_{i+1}\right)-f\left(x_{i-1}\right)}{x_{i+1}-x_{i-1}}
$$

then one would obtain:

$$
\left.\frac{\mathrm{df}}{\mathrm{dx}}\right|_{\mathrm{x}=\mathrm{x}_{\mathrm{i}}}=0
$$

at all points. Hence this mode can not be detected, and can grow unhindered. This means that this mode could grow to a level where it dominates the solution.

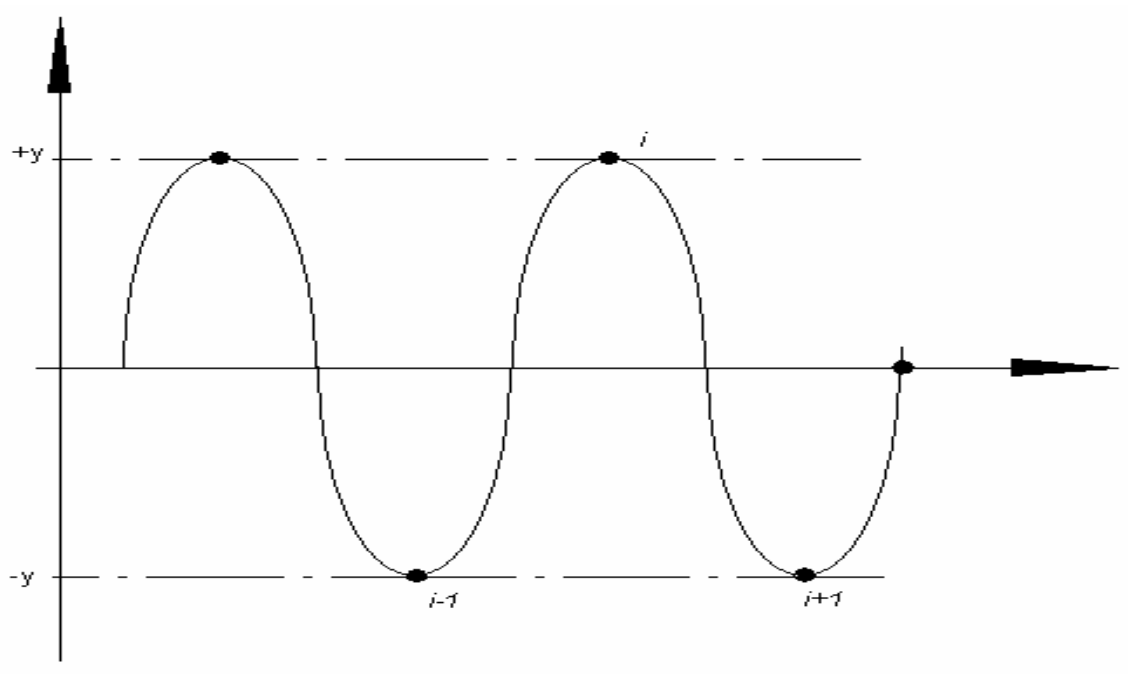

Figure 4: Zero energy modes.

Zero energy or spurious modes are characterised by a pattern of nodal displacement that is not a rigid body but produces zero strain energy.

One of the key ideas to reduce spurious oscillations is to compute derivatives away from the particles where kernel functions have zero derivatives. Randles [42] proposed a stress point method. Two sets of points are created for the domain discretisation, one carries velocity, and another carries stress. The velocity gradient and stress are computed on stress points, while stress divergence is sampled at the velocity points using stress point neighbours. According to [45], these spurious modes can be eliminated by replacing the strain measure by a non-local approximation based on gradient approach. Beissel [4] proposed another way to stabilise nodal integration, the least square stabilisation method.

\section{Summary}

The paper gives an overview of developments of the SPH method. Especial attention is given to the main shortcomings of the original form of the method 
namely consistency, tensile instability and zero energy modes. An example of derivation of correction necessary to assure first order consistency is given. The origin of the tensile instability and few proposed solutions to this problem are described. Similar consideration is given with respect to the zero energy modes typical for the collocational SPH method.

\section{References}

[1] Atluri S., T. Zhu. A new Meshless Local Petrov Galerkin (MLPG) approach in computational mechanics, Computational Mechanics, Vol 22, pp 117-127, 2000.

[2] Atluri S., H. G. Kim, J. Y. Cho, A critical assessment of the truly Meshless Local Petrov Galerkin (MLPG) and Local Boundary Integral Equation (LBIE), Computational Mechanics, Vol 24, pp 348-372, 2000.

[3] Attaway S. W., M.W. Heinstein, and J.W. Swegle. Coupling of smooth particle hydrodynamics with the finite element method. Nuclear Engineering and Design, Vol 150, pp199-205, 1994.

[4] Beissel, S. and Belytschko, T. Nodal integration of the element-free Galerkin method. Computational Methods Applied Mechanical Engineering, Vol. 139, pp. 49-71, 1996.

[5] Belytschko T., Y. Krongauz, D. Organ, M. Fleming, P. Krysl. Meshless Methods: An overview and recent developments Computer methods in applied mechanics and engineering, Vol 139, pp3-47, 1996.

[6] Belytschko T., Y.Y. Lu, and L. Gu. Element-free Galerkin methods. International Journal for Numerical methods in Engineering, Vol 37, pp229256, 1994.

[7] Belytschko T., Xiao S. Stability Analysis of Particle Methods with Corrected Derivatives, http://www.tam.northwestern.edu/xiaop/stable.html, 2002.

[8] Benz, W. Smooth particle hydrodynamics: a review. In J.R. Buchler, editor, The Numerical Modelling of Nonlinear Stellar Pulsatations, pp. 269-288. Kluwer Academic Publishers, 1990. Cited in: Campbell, J. Lagrangian hydrocode modelling of hypervelocity impact on spacecraft. $\mathrm{PhD}$ thesis, Cranfield University, 1998.

[9] Bonet, J. and Kulasegaram, S. Correction and stabilization of smooth particle hydrodynamics methods with application in metal forming simulation. International Journal of Numerical Methods Engineering, Vol. 47, pp. 1189 $1214,1999$.

[10] Campbell J., R. Vignjevic, L. Libersky. A Contact Algorithm for Smoothed Particle Hydrodynamics, Computer Methods in Applied Mechanics and Engineering, Vol. 184/1, pp 49-65, March 2000.

[11] Campbell P. M. Some new algorithms for boundary value problems in smooth particle hydrodynamics. Technical Report DNA-TR-88-286, Mission Research Corporation, 1989.

[12] Dilts G. A. Moving -least squares-particle hydrodynamics I, consistency and stability. Submitted to International Journal for Numerical Methods in Engineering, 1997. 
[13] Dyka C. T. and R.P. Ingel. An approach for tension instability in smoothed particle hydrodynamics (SPH). Computers and Structures, Vol 57(4), pp573580, 1995.

[14] Gingold, R.A. and Monaghan, J.J. Smoothed particle hydrodynamics: theory and application to non-spherical stars. Monthly Notices Royal Astronomical Society, Vol. 181, pp. 375-389, 1977.

[15] Guenther, C., Hicks, D.L., Swegle, J.W. Conservative smoothing versus artificial viscosity. Technical Report SAND94-1853, 1994.

[16] Harten A., P. D., Lax P. D., van Leer B. On upstream differencing and Godunov-type schemes for hyperbolic conservation laws, SIAM Review, Vol 25, no 1, pp 35-61, 1983.

[17] Hughes T. J. R. The finite element method, Prentice Hall, 1987.

[18] Johnson, G.R., Petersen, E.H., Stryk, R.A. Incorporation of an SPH option in the EPIC code for a wide range of high velocity impact computations. International Journal of Impact Engineering, Vol. 14, pp. 385-394, 1993.

[19] Johnson, G.R. Linking of Lagrangian particle methods to standard finite element methods for high velocity impact computations. Nuclear Engineering and Design, Vol. 150, pp. 265-274, 1994.

[20] Johnson G. R., S.R. Beissel. Normalised smoothing functions for SPH impact computations. International Journal for Numerical Methods in Engineering, Vol 39, pp2725-2741, 1996.

[21] Johnson, G.R., Stryk, R.A., Beissel, S.R. SPH for high velocity impact computations. Computer Methods in Applied Mechanics and Engineering, Vol. 139, pp. 347-373, 1996.

[22] Krongauz Y., Belytschko T. Enforcement of essential boundary conditions in meshless approximations using finite elements. Submitted to International Journal for Numerical Methods in Engineering, 1997.

[23] Krongauz Y., Belytschko T. Consistent pseudo derivatives in meshless methods. Computer methods in applied mechanics and engineering, Vol 146, pp371-386, 1997.

[24] Landau L. D., Lifshitz E.M. Mechanics, Course of Theoretical Physics, Vol 1, Pergamon Press, 1960.

[25] Lattanzo J. C., J.J. Monaghan, H. Pongracic, P. Schwarz. Controlling penetration. SIAM, Journal for Scientific and Statistic Computation, Vol 7, No 2, pp 591-598, 1996.

[26] Libersky, L.D., Petschek, A.G. Smooth particle hydrodynamics with strength of materials. Advances in the Free Lagrange Method, Lecture Notes in Physics, Vol. 395, pp. 248-257, 1990.

[27] Libersky, L.D., Petschek, A.G., Carney, T.C., Hipp, J.R., Allahdadi, F.A. High Strain Lagrangian Hydrodynamics: A Three-Dimensional SPH Code for Dynamic Material Response, Journal of Computational Physics, Vol. 109, Issue 1, November, pp. 67-75, 1993.

[28] Lucy, L.B. A numerical approach to the testing of fusion process. Astronomical Journal, Vol. 88, pp. 1013-1024, 1977.

[29] Meglicki, Z., Analysis and Application of Smoothed Particle Magnetohydrodynamics. PhD thesis, Australian National University. Cited in 
Campbell, J. Lagrangian hydrocode modelling of hypervelocity impact on spacecraft. Cranfield University, College of Aeronautics, PhD thesis, 1998.

[30] Mitchell A. R., Griffiths D. F. The finite difference method in partial differential equations, John Wiley, 1980.

[31] Monaghan J. J. Why particle methods work. SIAM Journal on Scientific and Statistical Computing, Vol 3(4), pp422-433, 1982.

[32] Monaghan, J. J., Gingold, R. A. Shock simulation by the particle method SPH. Journal of Computational Physics, Vol. 52, pp. 374-389, 1983.

[33] Monaghan, J. J., Lattanzio, J. C. A refined particle method for astrophysical problems. Astronomy and Astrophysics, Vol. 149, Issue 1, pp. 135-143, 1985.

[34] Monaghan J. J., Pongracic H. Artificial viscosity for particle methods. Applied Numerical Mathematics, Vol 1, pp187-194, 1985.

[35] Monaghan J. J. On the problem of penetration in particle methods. Journal of Computational Physics, Vol 82, pp1-15, 1989.

[36] Monaghan J. J. Smoothed Particle Hydrodynamics. Annual Review of Astronomy and Astrophysics, Vol 30, pp543--574, 1992.

[37] Monaghan, J. J. SPH without a Tensile Instability. Journal of Computational Physics, Vol. 159, pp. 290-311, 2002.

[38] Moussa B. Meshless Particle methods: Recent developments for non-linear conservation laws in bounded domain, in Godunov Methods: Theory and Applications, E. F. Toro (Editor), Kluwer Academic/Plenum Publishers, 2000.

[39] Moussa B., Vila J. P. Convergence of the SPH method for scalar nonlinear conservation laws, SIAM Journal of Numerical Analysis, Vol 37, No 3, pp 863-887, 2000.

[40] Petschek, A. G., Libersky, L. D. Cylindrical smoothed particle hydrodynamics. Journal of Computational Physics, Vol. 109, pp. 76-83, 1993.

[41] Randles P. W., Libersky L. D. Smoothed particle hydrodynamics: Some recent improvements and applications. Computer methods in applied mechanics and engineering, Vol 139, pp375-408, 1996.

[42] Randles, P. W., Libersky, L. D., Petschek, A. G. On neighbors, derivatives, and viscosity in particle codes, in: Proceedings of ECCM Conference, Munich, Germany, 31 August-3 September 1999.

[43] Resnyansky, A. D. DYNA-modelling of the high-velocity impact problems with a split-element algorithm, International Journal of Impact Engineering, Vol. 27, Issue 7, pp. 709-727, 2002.

[44] Stryk R. A., Johnson G. R., Beissel S. R. SPH for high velocity impact computations. Computer methods in applied mechanics and engineering, Vol 139, pp347-373, 1996.

[45] Swegle, J. W., Attaway, S. W., Heinstein, M. W., Mello, F. J., Hicks, D. L. An analysis of smooth particle hydrodynamics. Sandia Report SAND93-2513, 1994.

[46] Takeda H. T., Miyama S. M., Sekiya M. Numerical simulation of viscous flow by smooth particle hydrodynamics, Progress of Theoretical Physics, Vol 92 pp939-960, 1994.

[47] Toro E. F., A Linearised Riemann Solver for the time dependent Euler equations of gas dynamics, Proc. Roy. Soc. London, Vol A434, pp 683-693, 1991. 
[48] Toro E. F., Direct Riemann solvers for the time-dependent Euler equations, Shock Waves, Vol 5, pp 75-80, 1995.

[49] Toro E. F. Riemann Solvers and Numerical Methods for Fluid Dynamics, Second edition, Springer Verlag, 1999.

[50] Van der Vegt L. J. J. W., van der Ven H., and Boelens O. J. Discontinuous Galerkin methods for partial differential equations, in Godunov Methods: Theory and Applications, 1995.

[51] Vignjevic R., Campbell J., Libersky L. A Treatment of Zero Energy Modes in the Smoothed Particle Hydrodynamics Method, Computer Methods in Applied Mechanics and Engineering, Vol. 184/1, pp 67-85, 2000.

[52] Vignjevic, R., De Vuyst, T., Campbell, J. The use of a homogeneous repulsive force for contact treatment in SPH. Fifth World Congress on Computational Mechanics, Vienna, Austria, July 7-12, 2002.

[53] Vignjevic, R., Hughes, K. and Taylor E. A. Finite element modelling of failure of a multi-material target due to high velocity space debris impacts. Space Debris, Vol. 2, pp. 41-50, 2002.

[54] Vignjevic R., De Vuyst T., Gourma M. Interpolation Techniques in Meshless Methods, Computer Modelling in Engineering and Science Journal, Vol 2, No. 3, pp 319-337, 2001.

[55] Wen, Y., Hicks, D. L. and Swegle, J. W. Stabilising SPH with conservative smoothing. Technical Report SAND94-1932, 1994.

[56] Liu W. L., Jun S., Li S., Adee J., Belytschko T. Reproducing kernel particle methods for structural dynamics. International Journal for Numerical Methods in Engineering, Vol 38, pp1655-1679, 1995.

[57] Liu W. L., Jun S., Li S., Adee J., Belytschko T. Reproducing kernel particle methods for nearly incompressible hyperelastic solids. Submitted to International Journal for Numerical Methods in Engineering, 1997.

[58] Randles P. W., Libersky, L. D. Normalized SPH with Stresspoints. International Journal for Numerical Methods in Engineering, Vol. 48, pp. 1445-1462, 2000.

[59] Balsara D. P. Von Neumann Stability Analysis of Smoothed Particle Hydrodynamics - Suggestions for Optimal Algorithms. Journal of Computational Physics, Vol. 121, pp. 357-372, 1995.

\section{Bibliography}

Campbell, J., Vignjevic, R. Development of Lagrangian hydrocode modelling for debris impact damage prediction. International Journal of Impact Engineering, Vol. 20, pp. 143-152, 1997.

MCM A 1D/2D/3D Meshless Continuum Mechanic Code for solid and fluid mechanics. User Manual. School of Engineering, Cranfield University. Version 2.0. August 2002.

Campbell, J. Lagrangian hydrocode modelling of hypervelocity impact on spacecraft. PhD thesis, College of Aeronautics, Cranfield University, 1998. 